\title{
EphA3 functions are regulated by collaborating phosphotyrosine residues
}

\author{
Guanfang Shi ${ }^{1,3}$, Gang Yue ${ }^{2}$, Renping Zhou ${ }^{1}$ \\ ${ }^{I}$ Department of Chemical Biology, Ernest Mario School of Pharmacy, Rutgers University, Piscataway, NJ 08854, USA; \\ ${ }^{2}$ Department of Oral Biology, New Jersey Dental School, University of Medicine \& Dentistry of New Jersey, Newark, NJ 07101, \\ $U S A$
}

Ephrin ligands interact with Eph receptors to regulate a wide variety of biological and pathological processes. Recent studies have identified several downstream pathways that mediate the functions of these receptors. Activation of the receptors by ephrin binding results in the phosphorylation of the receptor tyrosine residues. These phosphorylated residues serve as docking sites for many of the downstream signaling pathways. However, the relative contributions of different phosphotyrosine residues remain undefined. In the present study, we mutated each individual tyrosine residues in the cytoplasmic domain of $\mathrm{EphA3}$ receptor and studied the effects using cell migration, process retraction, and growth cone collapse assays. Stimulation of the EphA3 receptor with ephrin-A5 inhibits 293A cell migration, reduces NG108-15 cell neurite outgrowth, and induces growth cone collapse in hippocampal neurons. Mutation of either Y602 or Y779 alone partially decreases EphA3-induced responses. Full abrogation can only be achieved with mutations of both Y602 and Y779. These observations suggest a collaborative model of different downstream pathways.

Keywords: tyrosine phosphorylation; signal transduction; cell migration; growth cone collapse Cell Research (2010) 20:1263-1275. doi:10.1038/cr.2010.115; published online 10 August 2010

\section{Introduction}

The Eph family tyrosine kinase receptors and their cognate ligands, the ephrins, play critical roles in embryonic development, postnatal growth, and pathogenesis [1-3]. Several Eph receptors and their ligands have been reported to regulate the formation of axon projection maps in the brain [4-6] and the segmentation of the hindbrain [7]. In addition, Eph receptors and their ligands regulate vascularization and angiogenesis $[8,9]$, and participate in bone morphogenesis $[10,11]$, inflammatory responses [12], lens development [13-15], neural plasticity modulation [16], and pathogenic processes such as tumorigenesis $[17,18]$. Downstream mechanisms medi-

Correspondence: Renping Zhou

Tel: +732-445-3400 x 264; Fax: +732-445-0687

E-mail: rzhou@rci.rutgers.edu

${ }^{3}$ Current address: Aab Cardiovascular Research Institute, University of Rochester Medical Center, Rochester, NY 14642, USA.

Received 5 April 2010; revised 31 May 2010; accepted 8 June 2010; published online 10 August 2010 ating Eph receptor functions have been actively investigated. Previous studies indicated that the cytoplasmic domain and kinase activity of Eph receptor are essential for many Eph receptor functions [19-24]. Upon ephrin binding, the tyrosine kinase activity of the Eph receptors becomes activated, which leads to the phosphorylation of the tyrosine residues in the cytoplasmic domain of the receptors [25]. Tyrosine phosphorylation of Eph receptors relays the extracellular signals into the cell upon ephrin binding by generating docking sites for downstream molecules [26]. For example, it has been shown previously that tyrosine 602 and 779 of EphA3 are phosphorylated and both are required to mediate cell de-adhesion upon ephrin-A5 stimulation [27]. Phosphorylation at tyrosines 605 and 611 activates EphB2 kinase activity, which leads to cell rounding, and both tyrosine residues are binding sites for the SH2 domain protein Src [28]. However, replacement of these two conserved tyrosine with glutamic acid prevents the binding of $\mathrm{SH} 2$ domain proteins without abrogating kinase activity and EphB2-mediated cell rounding $[28,29]$, indicating that other docking sites and interacting proteins may exist to carry out receptor 
functions. Kalo et al. [30] found that many tyrosine residues could be phosphorylated in the EphB2 cytoplasmic domain in retina tissue and 293A cells, including tyrosines $605,611,668,789$, and 939 , suggesting a model in which multiple binding sites and signaling pathways may act downstream of activated Eph receptors. Indeed, multiple signal transduction pathways have been shown to mediate functions of Eph receptors, including the Src family kinases [31-33], Rho family GTPase [34], cGMPdependent protein kinase $[33,35]$, and myosin light chain kinase [33]. In contrast to this wealth of information known about various downstream pathways, little is known about the relative contributions by these different pathways to specific Eph receptor functions.

To evaluate the relative contributions of different phosphotyrosine residues of Eph receptors in mediating downstream signaling functions, we performed a comprehensive analysis on the effects of tyrosine residue mutations in EphA3 cytoplasmic domain using several different biological assays. Our results show that the phosphorylation of the conserved juxtamembrane tyrosine residue Y602 and kinase domain tyrosine Y779 collaborate to induce growth cone collapse and inhibition of cell migration. These results confirm and extend results from previous studies and support a model in which different pathways docked at different phosphorylated tyrosine sites cooperate in mediating Eph receptor functions.

\section{Results}

Multiple tyrosine residues of EphA3 are phosphorylated

To reveal underlying mechanisms by which EphA3 receptor relays ephrin-A5 signals, we mutagenized tyrosine residues in the cytoplasmic domain of EphA3 and examined effects of mutations on the levels of receptor tyrosine phosphorylation. There are a total of 15 tyrosine residues in this domain, and each was mutated to either phenylalanine (F) or glutamic acid (E) (Table 1). Mutation of tyrosine into phenylalanine prevents phosphorylation of the residue, while mutation into glutamic acid adds a negative charge that mimics phosphorylated tyrosine [28]. The EphA3 mutants were transfected into HEK 293A cells, and 2 days later, the transfected cells were starved overnight in DMEM without any serum and lysed the next day. The total cell lysates were then analyzed for tyrosine phosphorylation using western blot with an antiphosphotyrosine-specific antibody. This analysis showed that the Y596F mutant exhibited a total loss of autophosphorylation, and so did the kinase-dead mu-

Table 1 Primers used in site-directed mutagenesis

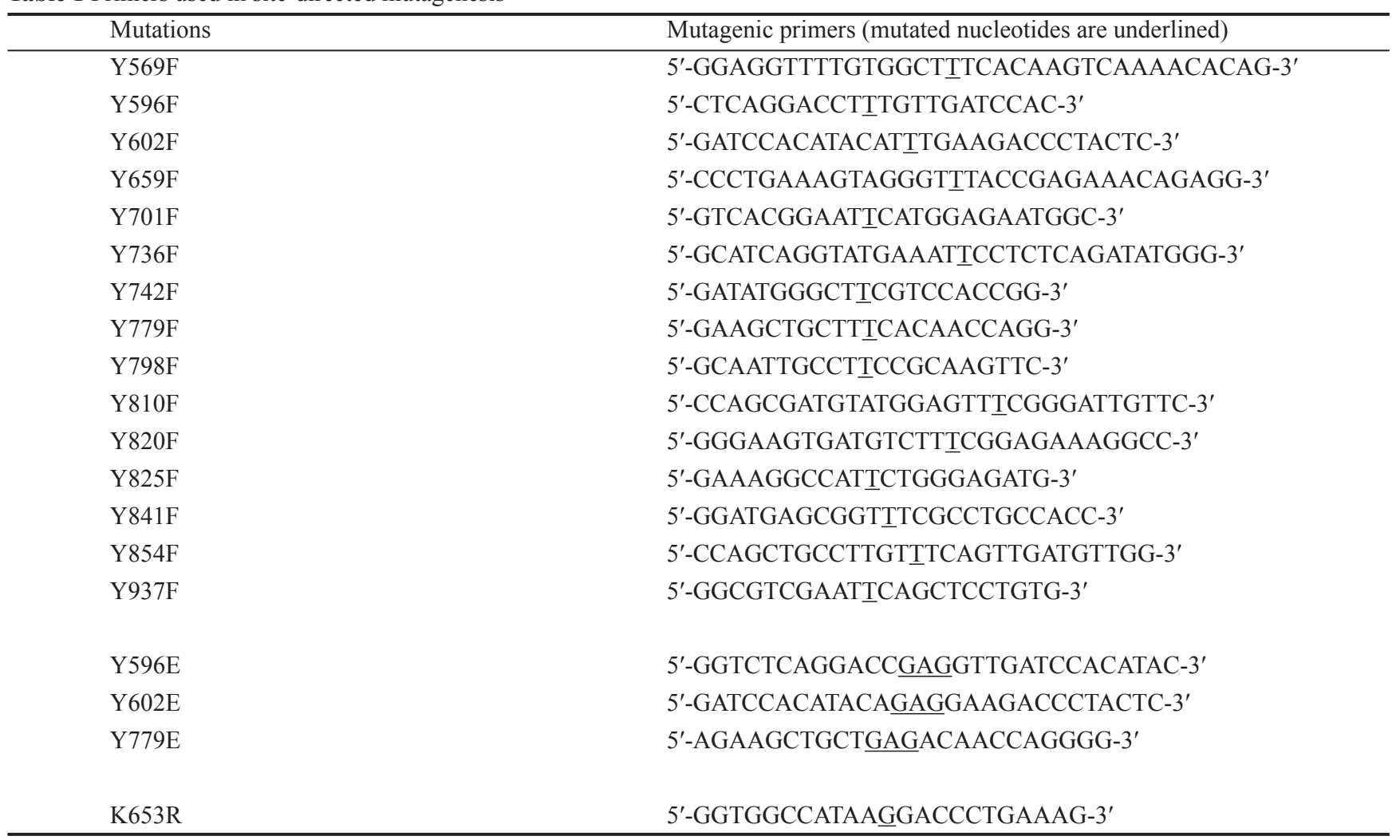


A

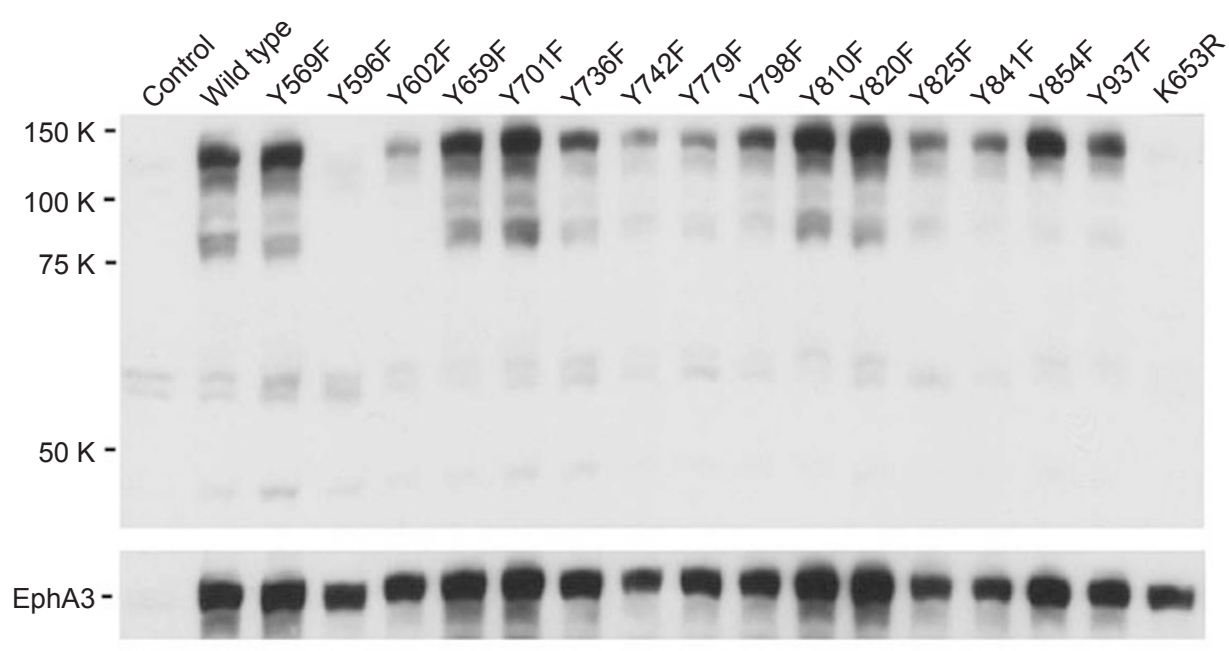

B

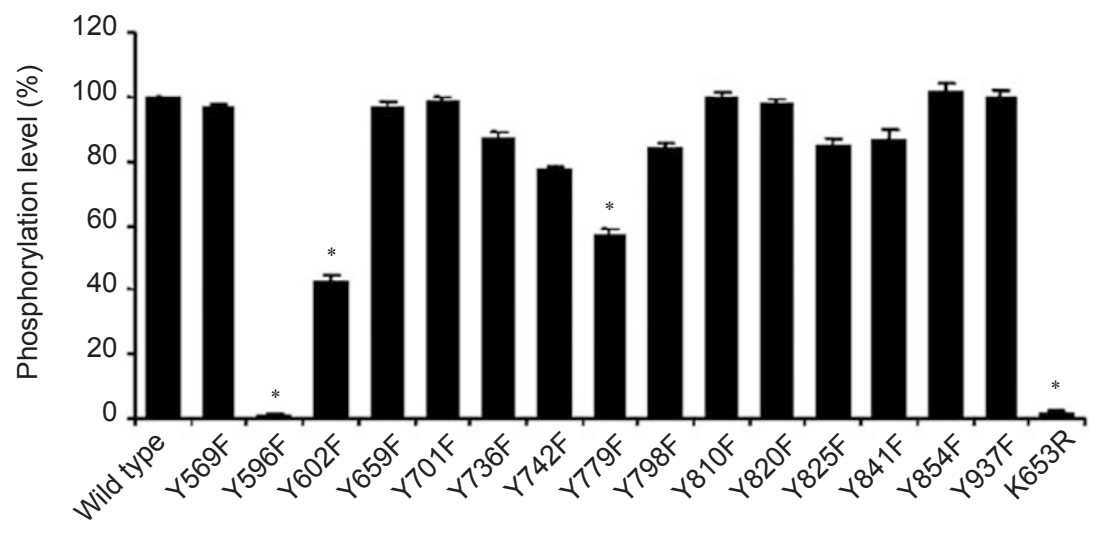

Figure 1 Effects of tyrosine mutations on EphA3 autophosphorylation. (A) Reduction of EphA3 autophosphorylation in several tyrosine mutants. EphA3 mutants were transfected into HEK 293A cells and treated with clustered ephrin-A5 for 15 min 2 days later. Cell lysates were analyzed by western blot analysis. Top panel: western blot analysis with antiphosphotyrosine antibody; Bottom panel: the same membrane was re-probed with an anti-EphA3 antibody to assess protein levels. (B) Quantification of the relative intensities of receptor autophosphorylation with optical intensity scanning. Data were normalized to cells harboring wild-type EphA3. Bars represent the means of three independent experiments; error bars indicate standard deviation. Asterisk '*' indicates $\alpha<0.05$ vs wild-type (one-way ANOVA followed with Bonferroni test for selected pairs).

tant K653R (Figure 1), suggesting a loss of kinase activity in these two mutants. Several other mutants, including Y602F, Y736F, Y742F, Y779F, Y798F, Y825F, Y841F, and $\mathrm{Y} 937 \mathrm{~F}$, all showed reduction in autophosphorylation levels (Figure 1). However, Y602F and Y779F showed more substantial decreases than the other mutants except Y596F and K653R (Figure 1B). These observations indicate that multiple tyrosines are phosphorylated and tyrosine 596 may regulate EphA3 kinase activity.

\section{Phosphorylation of Y596 is crucial for EphA3 tyrosine kinase activity}

The decrease in tyrosine phosphorylation of EphA3 mutants could be due either to the loss of kinase activity or phosphorylation sites. To differentiate between these two possibilities, we assayed the kinase activity using enolase as a substrate. This analysis showed that Y596F, like the kinase-dead mutant K653R, did not have any detectable kinase activity, while all other tyrosine mutants displayed normal enzymatic activity (Figure 2A). Phosphorylation of the juxtamembrane domain tyrosine residues has been shown to play key roles in releasing intramolecular inhibition of the Eph receptor kinases [36]. To study whether Y596 phosphorylation is required for EphA3 activation, we replaced this tyrosine residue with glutamic acid and assayed for effects on the kinase activity. The negative charge of glutamic acid residue in the juxtamembrane region has been shown to mimic that of 

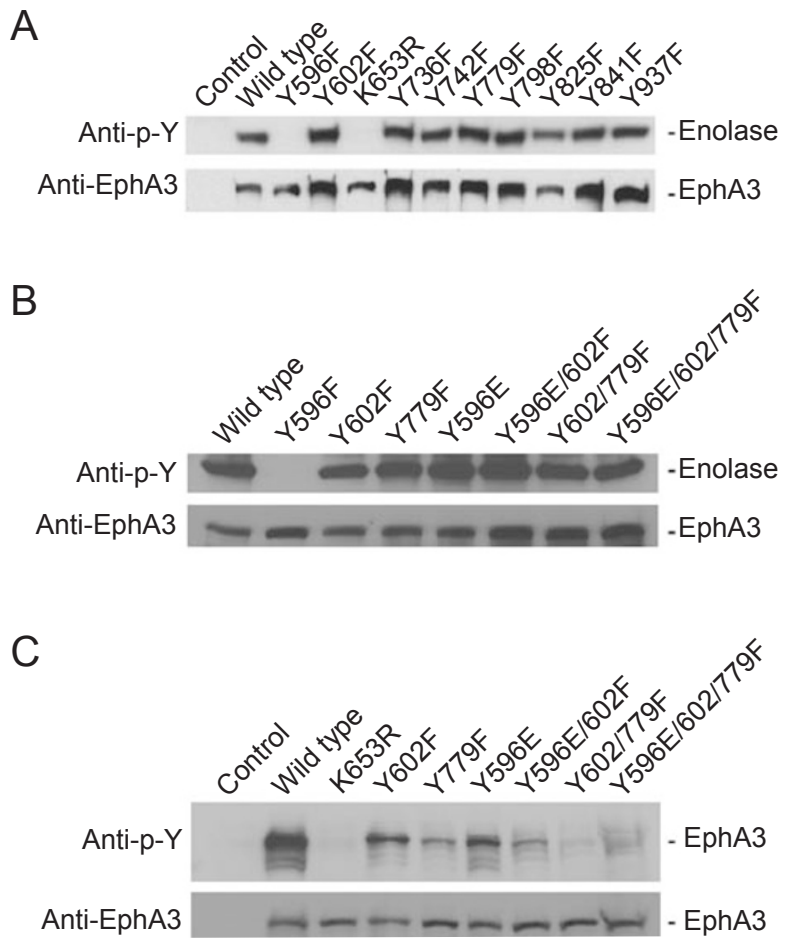

Figure 2 Tyrosine kinase activity of various EphA3 mutants. (A) Effects of the tyrosine to phenylalanine mutations on EphA3 kinase activity. EphA3 was immunoprecipitated from transfected 293A cells and incubated with $10 \mu \mathrm{g}$ of acid-denatured Enolase. The reaction was stopped by adding SDS sample-loading buffer and boiling. The reaction products were analyzed by immunoblotting with antiphosphotyrosine antibody (upper panel) and antiEphA3 antibody (lower panel), respectively. (B) Tyrosine kinase activity of the double and triple EphA3 mutants. (C) Receptor autophosphorylation levels in the double and triple EphA3 mutants.

phosphorylated tyrosine residues [28]. Replacement of Y596 with a glutamic acid residue completely restored EphA3 kinase activity (Figure 2B), suggesting that the negative charge on Y596 is crucial in activating EphA3. In contrast, phosphorylation of Y602 and Y779 is not required for EphA3 kinase activity, since Y602F, Y779F, the double mutants $\mathrm{Y} 596 \mathrm{E} / 602 \mathrm{~F}$ and $\mathrm{Y} 602 / 779 \mathrm{~F}$, as well as the triple mutant $\mathrm{Y} 596 \mathrm{E} / 602 / 779 \mathrm{~F}$ all possessed normal kinase activity (Figure 2B). Although Y602 and Y779 were not required for kinase activation, Y602F and $\mathrm{Y779F}$ mutations had significantly reduced levels of receptor tyrosine phosphorylation (Figures 1 and 2C), indicating that these two tyrosine residues were major EphA3 phosphorylation sites. In addition, although Y596E mutant was fully active in phosphorylating enolase, the receptor tyrosine phosphorylation was also significantly reduced (Figure 2C), suggesting that Y596 was another major phosphorylation site. Consistent with these conclusions, tyrosine phosphorylation signals of the double mutants Y596E/602F and Y602/779F and the triple mutant Y596E/602/779F were barely detectable (Figure $2 \mathrm{C})$. Taken together, these studies provide evidence that Y596 is required for EphA3 activation, and together with Y602 and Y779, these three tyrosine residues are major autophosphorylation sites.

Specific tyrosine residues of EphA3 are required for ephrin-A5-induced inhibition of cell migration

Previous studies have shown that activation of Eph receptors leads to inhibition of vascular smooth muscle cell spreading and COS cell migration $[37,38]$. To examine effects of specific tyrosine mutations, the mutants were transfected into HEK 293A cells and assayed for their effects on cell migration in the presence of ephrin-A5 using would-healing and transwell assays (Figure 3). Transfection of wild-type EphA3 resulted in efficient inhibition of cell migration by ephrin-A5 in both assays (Figure 3). In contrast, ephrin-A5 had no effects on the migration of cells transfected with the kinase-dead mutant K653R (Figure 3). Furthermore, our analyses showed that substitution of juxtamembrane tyrosine 596 with phenylalanine completely abolished effects on cell migration, while two other mutations, Y602F and $\mathrm{Y} 779 \mathrm{~F}$, resulted in partial loss of the function in both assays (Figure $3 \mathrm{~B}$ and $3 \mathrm{C}$ ). Mutations in other tyrosine residues did not have any obvious effects (Figure 3B and 3C). These results indicate that both the tyrosine kinase activity and the phosphorylation of Y602 and Y779 are crucial for the inhibition of cell migration.

Cooperative regulation of cell migration by Y602 and Y779

Since Y602F and Y779F mutants each partially reduced EphA3-mediated inhibition of cell migration compared to the wild type (Figure 3B and 3C), we asked whether these two tyrosine residues cooperatively regulate cell migration. Indeed, double EphA3 mutant Y602/779F lost all activity in mediating inhibition of cell migration in both the wound-healing and transwell assays (Figure 4). Similarly, the triple mutant Y596E/602/779F also lost all activity in mediating inhibition of cell migration. In contrast, the double mutant Y596E/602F had similar activity as that of the Y602F single mutant (Figures 3 and 4), which was consistent with the idea that phosphorylation of Y596 merely serves to activate the receptor kinase. These observations support the notion that Y602 and Y779 provide docking sites for downstream signal transduction pathways that together regulate cell migration. 
A

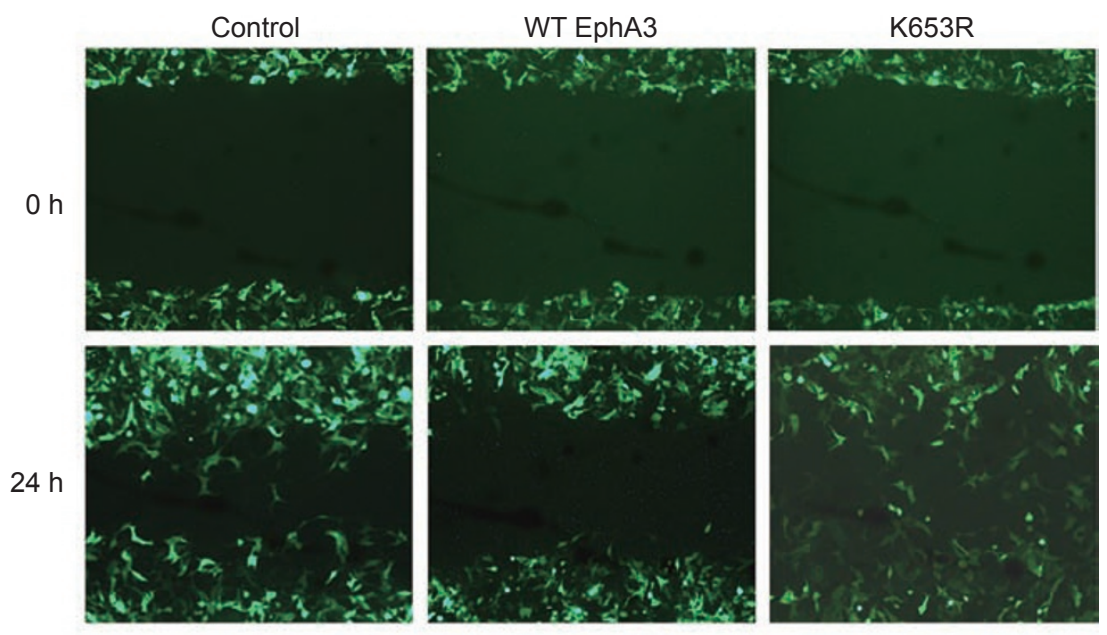

B

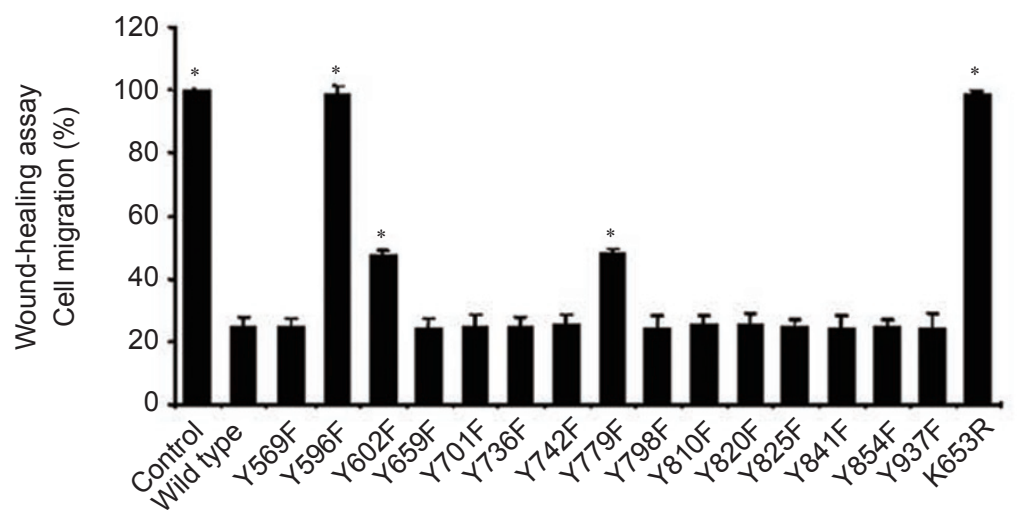

C

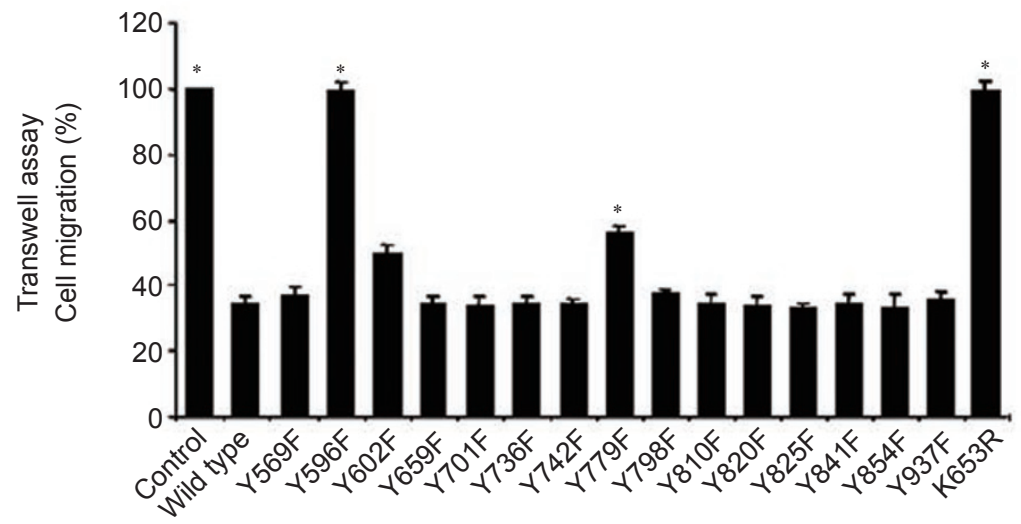

Figure 3 Phosphorylation of both Y602 and Y779 contributes to the inhibition of cell migration by EphA3. (A) Inhibition of cell migration by EphA3 is kinase dependent. 293A cells were transfected with wild-type EphA3, kinase-dead mutant K653R, or pCMV vector only, and assessed for effects on cell migration using the wound-healing assay. Images were taken at 0 and $24 \mathrm{~h}$ after wounding. (B) Quantification of effects of EphA3 tyrosine mutants on cell migration in the wound-healing assay. (C) Quantification of effects of EphA3 tyrosine mutations on cell migration in the Transwell assay. In both assays, cell counts were normalized to that of the vector controls. Bars represent the means of six independent experiments. Error bars indicate standard deviation. Asterisk '*' indicates $\alpha<0.05$ vs wild-type (WT) (one-way ANOVA followed with Bonferroni test for selected pairs). 
A

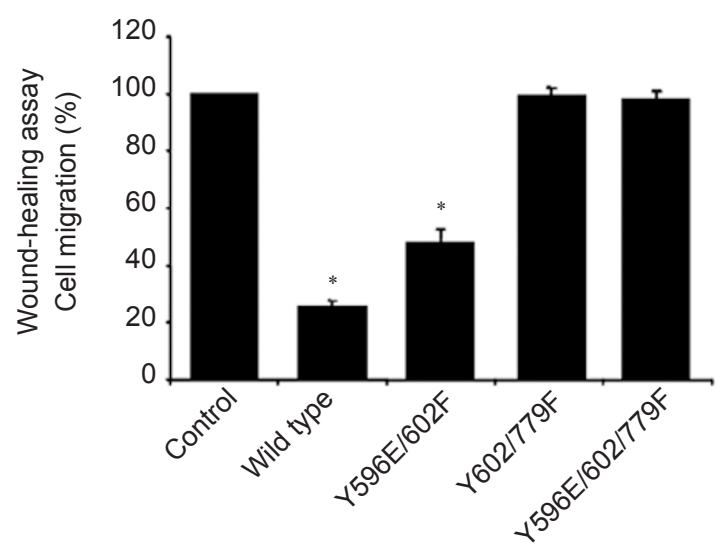

B

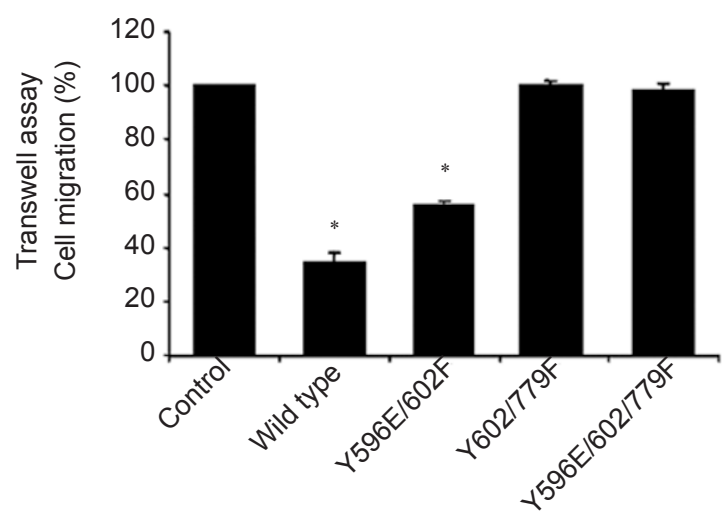

Figure 4 Effects of the double and triple EphA3 mutants on cell migration. Double and triple EphA3 mutants were similarly assayed as described in the Figure 3 legend. (A) Wound-healing assay. (B) Transwell assay. Cell counts are normalized to that of the vector controls. Bars represent means from six independent experiments; error bars represent SD. Asterisk '*' indicates $\alpha<$ 0.05 vs vector control (one-way ANOVA followed with Bonferroni test for selected pairs).

Activated EphA3 inhibits neurite outgrowth of transfected NG108-15 cells, and mutation at Y602 or Y779 results in partial relief from the inhibition

Eph receptors are involved in many different biological processes, and several signaling pathways have been implicated to carry out Eph functions. It is possible that different phosphorylated tyrosines may play different roles for different functions. To investigate this possibility, we have expressed wild-type and mutant EphA3 in NG108-15 neuronal cells. NG108-15 cell is a hybridomal cell line of a rat neuroblastoma and a mouse glioma [39]. With the stimulation of cyclic AMP, NG108-15 cells differentiated to a neuron-like phenotype with neurites (Figure 5). Transfection of EphA3 cDNAs followed by ephrin-A5 stimulation and western blot analysis showed that there is no detectable endogenous EphA3 expression and that the transfected genes can be expressed properly (Supplementary information, Figure S1). NG108-15 neurites disappeared when wild-type EphA3-transfected cells were treated with ephrin-A5, whereas cells transfected with the nonfunctional EphA3 mutants (K653R, Y596F) retained these neurites (Figure 5). To quantify the data, the density of neurites was normalized to that of the cell body staining. The quantification showed that both $\mathrm{Y} 602 \mathrm{~F}$ and $\mathrm{Y} 779 \mathrm{~F}$ mutants led to partial reduction of neurites, while double mutation Y602/779F completely abrogated the inhibition by EphA3 activation (Figure $5 \mathrm{~B})$, suggesting that the cooperation between these two tyrosine residues is also required for the inhibition of neurite outgrowth by ephrin-A5.

Ephrin-A5 induces growth cone collapse in EphA3transfected lateral hippocampal neurons

Previous reports have shown that EphA3 is highly expressed in the medial hippocampus and its expression is low in the lateral regions [23]. To examine whether neurons from different regions of the hippocampus responded to ephrin-A5 treatment similarly, rat hippocampus was dissect into medial and lateral portions and cultured separately. As expected, ephrin-A5 treatment led to significant growth cone collapse in neurons from medial hippocampus rather than those from lateral hippocampus (Figure 6A and 6B). Thus, the lateral hippocampal neurons were used to assay the effects of mutations on EphA3 function in growth cone collapse assay.

We first determined whether the hippocampal neurons could be transfected efficiently. Hippocampal neurons were transfected with wild type and mutant EphA3, and analyzed for EphA3 expression after 2 days of culture. This analysis showed that exogenous EphA3 can be expressed effectively in the hippocampal neurons (Supplementary information, Figure S2). Treatment of the transfected neurons with ephrin-A5 showed that the transfection of wild-type EphA3 into medial hippocampal neurons did not increase the number of collapsed growth cones, while kinase-dead EphA3 worked as a dominantnegative mutant that rescued growth cones from collapse (Figure 6C). The inhibition on neurite growth by EphA3 activation was further supported by EphA3 transfection into the lateral hippocampal neurons, leading to a dramatic response to ephrin-A5, as compared to those harboring control vectors (Figure 6D).

EphA3-mediated growth cone collapse requires the phosphorylation of both Y602 and Y779 to achieve the full effect

To examine the relative contributions of Y602 and Y779 in EphA3-mediated growth cone collapse, lateral 
A
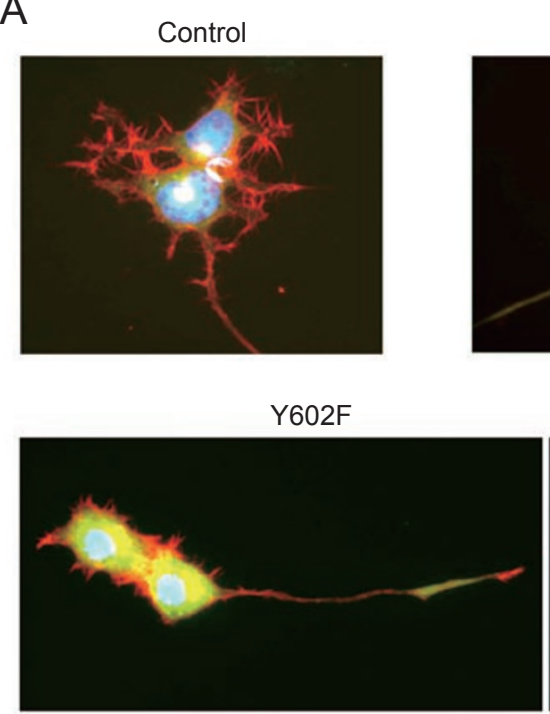

WT EphA3

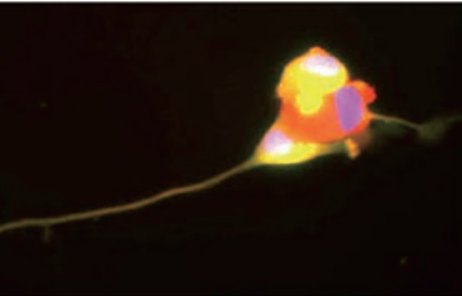

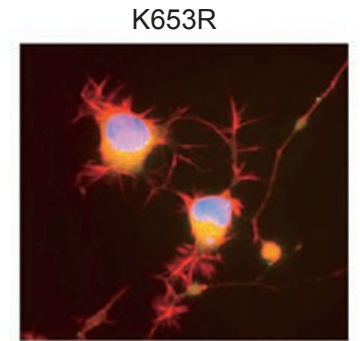

Y779F

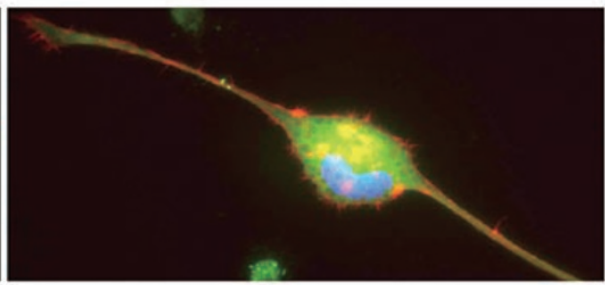

Y602/779F

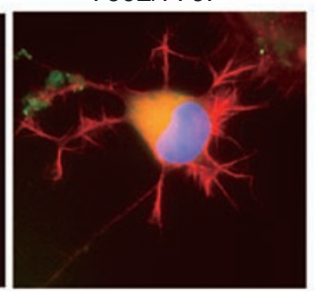

Y596F

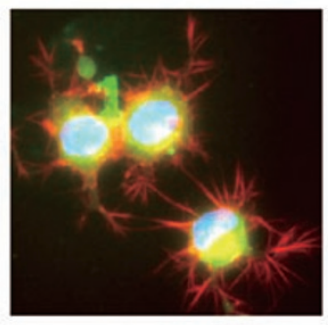

B

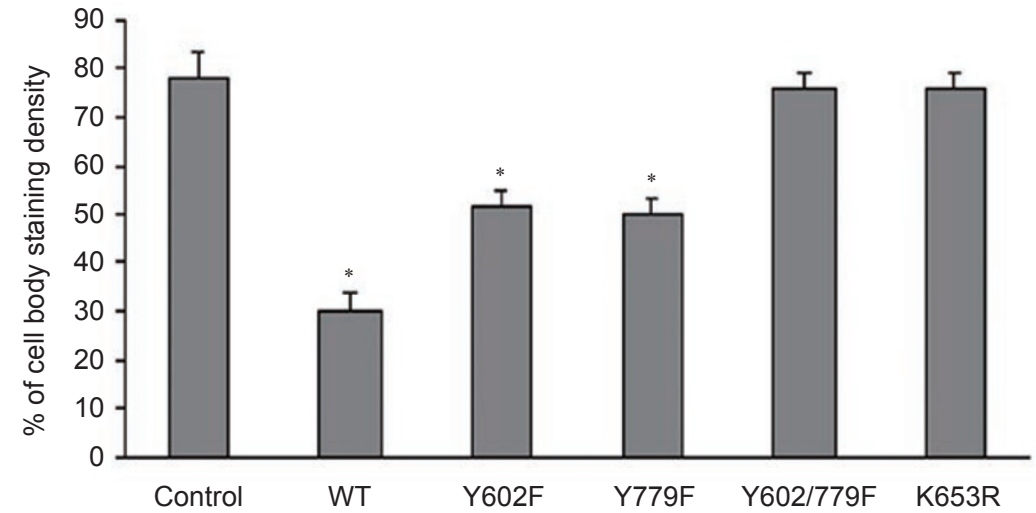

Figure 5 EphA3 inhibits formation of neurites in transfected NG108-15 cells. (A) NG108-15 cells were transfected with EphA3 mutants, differentiated, and treated with $2 \mu \mathrm{g} / \mathrm{ml}$ cross-linked ephrin-A5 overnight. Cells were fixed with $4 \%$ paraformaldehyde in PBS and stained with Texas Red-conjugated phalloidin. Neurite outgrowth in NG108-15 cells was observed and recorded with a Nikon fluorescence microscope. (B) Quantification of the relative intensity of neurite staining. Data were normalized to cell body staining. Bars represent the means of at least 15 cells from three independent experiments; error bars indicate standard deviation. Asterisk '*' indicates $\alpha<0.05$ vs control (one-way ANOVA followed with Bonferroni test for selected pairs).

hippocampal neurons were transfected with either wildtype or mutant EphA3 and treated with ephrin-A5. Both Y $602 \mathrm{~F}$ and $\mathrm{Y} 779 \mathrm{~F}$ led to a $20 \%$ reduction of growth cones, as compared to the control without EphA3 transfection, while wild-type EphA3-transfected cells showed more than $50 \%$ decrease in growth cones under ephrinA5 stimulation (Figure 7B), indicating a partial loss of function by each mutant. Complete abrogation of EphA3 function was achieved by the mutation of both Y 602 and Y779, similar to that of the kinase inactive mutants K653R and Y596F (Figure 7). Notably, the neurons with control vectors also showed a $10 \%$ decrease in growth cone count when ephrin-A5 is present, indicating a low- level expression of ephrin-A5 receptors, which was consistent with the previous report that EphA receptors are expressed in lateral hippocampus though at a lower level [23].

\section{Discussion}

In this study, we have assessed roles of different tyrosine residues of EphA3 in the regulation of kinase activity, cell migration, neurite outgrowth, and growth cone collapse. In particular, these studies identified two distinct tyrosine residues that cooperate to regulate these functions. 
A

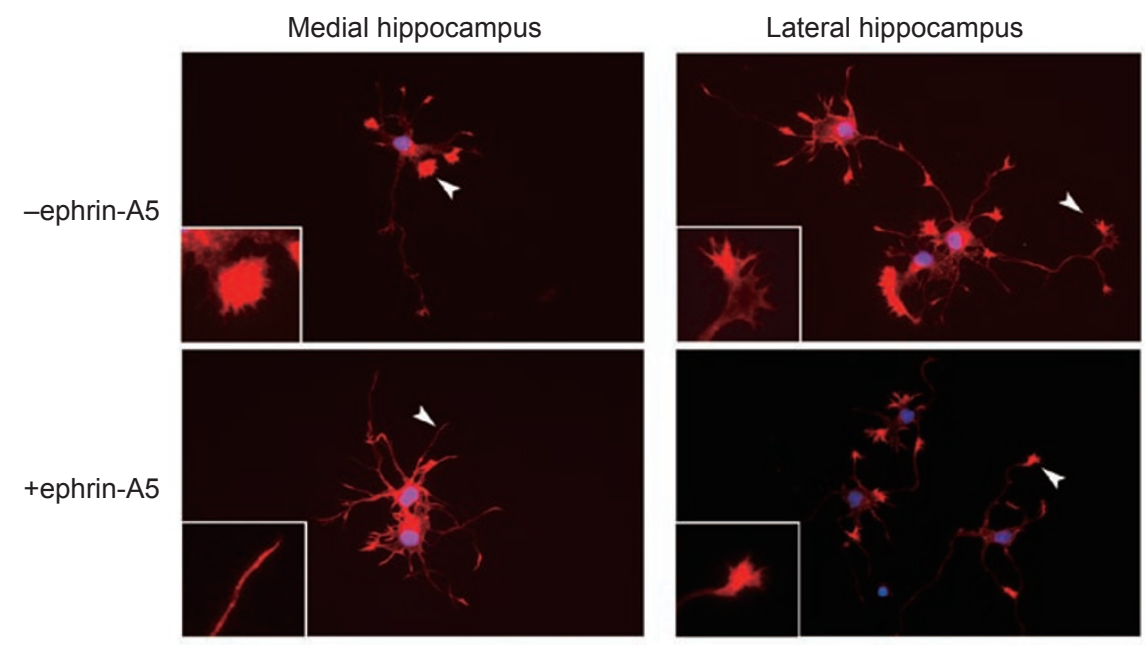

B

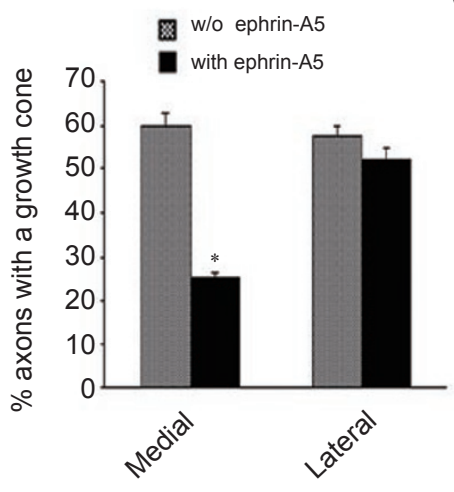

C

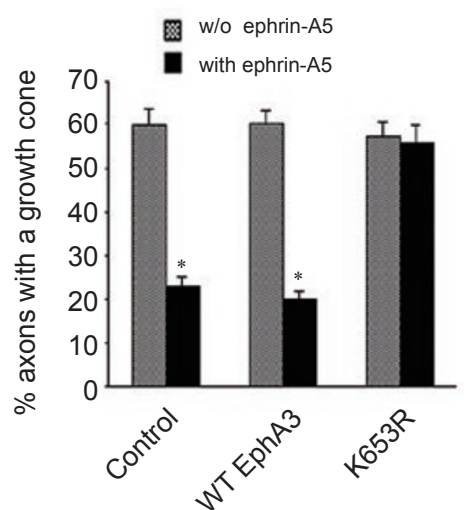

$\mathrm{D}$

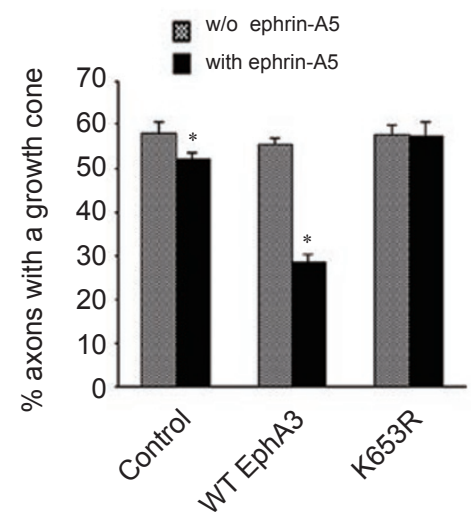

Figure 6 Medial and lateral hippocampal neurons respond to ephrin-A5 treatment differently. (A) Hippocampal neurons from rat E17 embryos were culture in neurobasal media for $48 \mathrm{~h}$. The cultures were treated with $2 \mu \mathrm{g} / \mathrm{ml}$ clustered ephrin-A5 for $15 \mathrm{~min}$ and stained for F-actin with Texas Red-conjugated phalloidin. The growth cone morphology was examined under a Zeiss fluorescence microscope. Arrowheads indicate representative growth cones shown in inserts. (B) Ephrin-A5 induces significant growth cone collapse in medial but not lateral hippocampal neurons. The number of the total axons and axons with a growth cone were enumerated, expressed as the percentage of axons that contain growth cones. Bars represent the means of at least 300 axons from three independent experiments; error bars indicate standard deviation. Asterisk '*' indicates $\alpha<0.05$ vs culture without ephrin-A5 treatment (one-way ANOVA followed with Bonferroni test for selected pairs). (C) Medial hippocampal neurons were transfected with wild-type or kinase-dead EphA3 plus GFP and treated with or without ephrin-A5. Growth collapse was quantified as in B. Only green cells were counted. (D) Lateral hippocampal neurons were transfected with wild-type or kinase-dead EphA3 plus GFP and treated with or without ephrin-A5. Growth collapse was quantified as in B. Only green cells were counted.

Our analyses also show that phosphorylation of the juxtamembrane tyrosine 596 is necessary for EphA3 activation. Replacing Y596 with phenylalanine inactivates EphA3. Both autophosphorylation assay and tyrosine kinase activity assay using the exogenous substrate enolase yielded results that are consistent with this conclusion (Figures 1 and 2). In further support of this conclusion, the tyrosine kinase activity is fully restored by a glutamic acid replacement in the tyrosine 596 mutant Y596E (Figure 2), which mimics the negative charge of phosphorylated tyrosine at this position [28]. The Y596E mutant displayed a relatively weaker tyrosine autophosphorylation of EphA3 receptor than the wild type, suggesting that Y596 is one of the major phosphorylation sites (Figure 2). However, a study by Lawrenson et al. [27] reported no loss of tyrosine kinase activity when ty- 
A
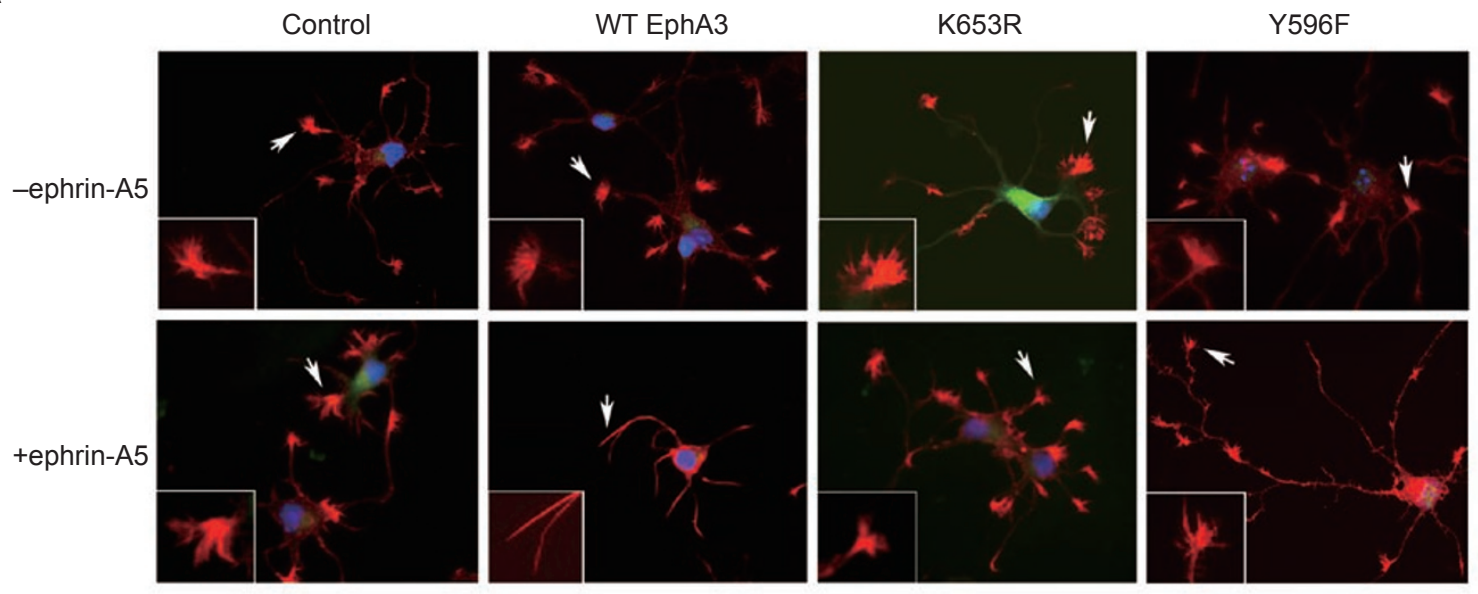

B

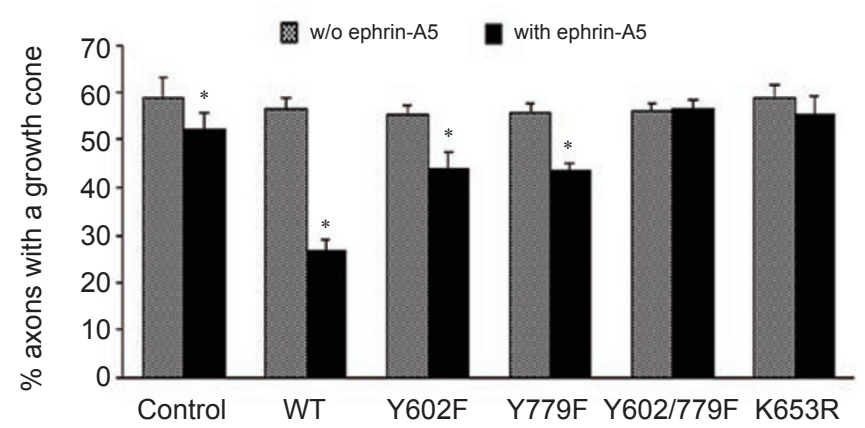

Figure 7 Effects of EphA3 mutations on growth cone collapse in lateral hippocampal neurons upon ephrin-A5 binding. (A) Lateral hippocampal neurons were transfected with wild-type or kinase-dead EphA3 plus GFP and treated with or without ephrin-A5. The growth cone morphology was examined under a Zeiss fluorescence microscope. Arrowheads indicate representative growth cones shown in inserts. Only transfected (GFP-positive) cells were included in the analysis. (B) Quantification of growth cone collapse in EphA3 mutant-transfected lateral hippocampal neurons. Bars represent the means of at least 300 axons from three independent experiments; error bars indicate standard deviation. Asterisk '*' indicates $\alpha<0.05$ vs culture without ephrin-A5 treatment (one-way ANOVA followed with Bonferroni test for selected pairs).

rosine 596 is mutated to phenylalanine in human EphA3, as measured by autophosphorylation. The cause of this discrepancy is not known at present, which could be due to sequence variations between these two molecules. A critical role of the juxtamembrane domain tyrosine residues in regulating the kinase activity is supported by studies of other Eph receptors [28]. Mutagenesis of the corresponding tyrosine at position 605 of EphB2 (corresponding to Y596 in EphA3) resulted in a reduction in both the kinase activity and autophosphorylation levels [28], consistent with our observations reported here. Interestingly, mutation of a second juxtamembrane region tyrosine of EphB2 (residue 611, corresponding to Y602 in EphA3) also reduces the kinase activity. Mutation of both residues led to complete inactivation of EphB2 kinase activity [28]. In contrast to EphB2, muta- tion of Y602 in EphA3 (corresponding to tyrosine 611 in EphB2) does not affect tyrosine kinase activity (Figure 2). A more recent study provided new evidence to support our conclusion that Y596 is important for kinase activity and Y602 acts as a docking site [40]. In this study, Davis et al. [40] reported that Y602 and its neighboring residues are solvent exposed instead of packed inside, as in the case of EphB2, suggesting that Y602 functions differently. Thus, although some variations exist among different Eph receptors, phosphorylation of the juxtamembrane tyrosine residues is necessary for the activation of the Eph receptors.

The current study identified two cytoplasmic tyrosine residues of EphA3 that together regulate cell migration, neurite outgrowth, and growth cone collapse, namely, Y602 and Y779. Mutation of either of these two residues 
alone leads to a partial reduction in EphA3 biological activity (Figures 1, 3, and 7). Mutation of both residues leads to a complete loss of biological activity, although the tyrosine kinase activity of the receptor remains intact (Figures 2, 3, and 7). Phosphorylated tyrosine residues have been known to serve as docking sites for SH2domain-harboring proteins to allow transduction of signals downstream [27]. Since the kinase activity in these mutants was not altered (Figure 2), deficiency in EphA3 activity is most likely due to a loss of phosphotyrosine docking sites that link with downstream signaling pathways.

A previous study by Lawrenson et al. [27] also identified Y602 and Y779 as required in a cell-rounding assay. In the absence of either Y602 or Y779, EphA3 receptor completely loses its ability to induce cell rounding. Our present study confirms the importance of these two tyrosine residues. In addition, the current study extended the previous analysis in several important aspects. First, the assays used in this study better correlate with cellular behavior in vivo. The wound-healing and transwell assays directly measure cell migration, a ubiquitous process during embryogenesis and cancer formation. The neurite outgrowth and growth cone collapse assays mimic behavior of growing neurites when they encounter negative guidance cues in the developing nervous system or during axon regeneration [41]. Although very useful, the cell-rounding assay used previously did not easily conform with behavior in vivo. Second, we showed here that tyrosine residues other than 596, 602, and 779 are not required for the inhibition of cell migration, process retraction, and growth cone collapse, although it is possible that they may play roles in other biological processes. Third, only a partial loss of EphA3 function was observed when a single mutation was introduced. This difference may be due to a higher sensitivity of the assays used in this study, which allows detection of more subtle differences. These observations suggest that different downstream pathways coupled to different phosphotyrosine residues collaborate together to mediate EphA3 functions.

Several different SH2-domain-containing proteins have been shown to bind to the corresponding juxtamembrane domain tyrosine residues on Eph receptors, including RasGAP, Src, Nck, and Vav [42-45]. Among these proteins, RasGAP has been shown to inhibit R-ras activity and regulate cell migration and growth cone collapse $[38,46,47]$. Src family kinases are also required for growth cone collapse [31-33]. In addition, our previously published study showed that Nck1 binds to phosphorylated Y602 directly and is involved in the regulation of cell migration [44]. Nck has also been shown to inter- act strongly with R-Ras proline-rich region [48], even though the role of Nck in R-Ras mediated integrin activation is still not clear [49]. Thus, the inhibitory effects mediated by Y602 may be due to the interaction with RasGAP, Src, Nck1, or Vav proteins.

The phosphorylated Y779 has been shown to bind to SH2-domain-containing Crk adaptor protein [27]. Interaction of Crk with Y779 is required for cell-rounding effects induced by EphA3 activation [27], and RhoA activation depends on Crk activity [50]. Activation of the small GTPase RhoA is a downstream event required for growth cone collapse induced by ephrin-A5 through the activation of EphA receptors [51]. Therefore, the partial loss of activity of EphA3 mutant Y779F may be due to the inability to activate RhoA. Taken together, our studies show that multiple pathways coupled to Y602 and Y779 contribute to EphA3-mediated regulation of cell migration and axon guidance. Since EphA3 mutations are found in several different types of human cancer [5255], information from the present study may also contribute to the understanding of mechanism of tumorigenesis.

\section{Materials and Methods}

\section{Site-directed mutagenesis}

Mouse EphA3 cDNA was a gift from Dr Elena B Pasquale. Desired mutations were generated using a Site-Directed Mutagenesis kit (BD Bioscience, Palo Alto, CA, USA) following the instructions of the manufacturer, and confirmed by DNA sequence analysis. The primers used in the mutagenesis are listed in Table 1.

\section{Cell culture and transfection}

HEK 293A cells were cultured in Dulbecco's modified Eagle's medium (DMEM) containing 10\% fetal bovine serum (FBS) at $37{ }^{\circ} \mathrm{C}$. NG108-15 cells were cultured in DMEM containing $1 \times$ HAT supplement $(0.1 \mathrm{mM}$ hypoxanthine, $400 \mathrm{nM}$ aminopterin, $0.016 \mathrm{mM}$ thymidine, Invitrogen, Carlsbad, CA, USA) and $10 \%$ FBS at $37{ }^{\circ} \mathrm{C}$. DNA transfection was performed using Lipofectamine 2000 (Invitrogen) following manufacturer's instructions.

Primary culture of rat hippocampal neurons and neuron transfection

Rat hippocampus was dissected out from rat E17 embryo. When needed, the hippocampus was further cut into two pieces: $2 / 3$ medial piece and 1/3 lateral piece. The dissected tissues were treated with $1 \times$ trypsin-EDTA solution at $37{ }^{\circ} \mathrm{C}$ for $15 \mathrm{~min}$ and then $1 \times$ trypsin inhibitor solution (Invitrogen) at room temperature for $15 \mathrm{~min}$. Neurons were dissociated by pipetting up and down several times and $2 \times 10^{5}$ neurons were seeded on $12-\mathrm{mm}$ polyD-lysine and laminin-coated cover slips for growth cone collapse study. Dissociated neurons were transfected by electroporation using the Nucleofector device and the Nucleofector Rat Neuron Kit (Amaxa, Gaithersburg, MD, USA), and then seeded on cover slips. Neurons were cultured in neural basal medium plus B27 supplement (Invitrogen) at $37^{\circ} \mathrm{C}$. GFP-expressing vectors were cotrans- 
fected to indicate the success of transfection.

\section{Ephrin-A5 preparation and treatment}

Ephrin-A5-Fc fusion protein was purchased from R\&D Systems (Minneapolis, MN, USA). To form clustered ephrin-A5, ephrin-A5-Fc was cross-linked with anti-human Fc IgG (Jackson Immuno-Research, West Grove, PA, USA) at 5:1 ratio in $\mu$ gs for $2 \mathrm{~h}$ at $37^{\circ} \mathrm{C}$ as described in our previous studies [33]. Ephrin-A5 treatment was carried out at $37^{\circ} \mathrm{C}$ for $15 \mathrm{~min}$.

\section{Immunoprecipitation and in vitro kinase assay}

Transfected HEK 293A cells were lysed with cell lysis buffer ( 50 $\mathrm{mM}$ Tris- $\mathrm{HCl}(\mathrm{pH} 8.0), 150 \mathrm{mM} \mathrm{NaCl}, 1 \% \mathrm{NP}-40,1 \mathrm{mM}$ sodium vanadate, and protease inhibitors). Cell lysates were then centrifuged to clear off cell debris for $10 \mathrm{~min}$ at $16000 \times \mathrm{g}$ at $4{ }^{\circ} \mathrm{C}$. The supernatant was incubated with a rabbit polyclonal anti-EphA3 antibody (Santa Cruz Biotechnology Inc, Santa Cruz, CA, USA) for $2 \mathrm{~h}$ and then with protein A agarose (Millipore, Billerica, MA, USA) for $1 \mathrm{~h}$ at $4{ }^{\circ} \mathrm{C}$. The beads were collected and washed three times with HNTG buffer (20 mM HEPES (pH 7.5), $150 \mathrm{mM} \mathrm{NaCl}, 10 \%$ glycerol, and $0.1 \%$ Triton X-100) and then twice with kinase buffer (25 mM HEPES (pH 7.5), $25 \mathrm{mM} \mathrm{MgCl}_{2}, 10 \mathrm{mM} \mathrm{MnCl}_{2}$, and $1 \mathrm{mM}$ sodium vanadate). Samples were incubated in kinase buffer containing $10 \mu \mathrm{g}$ of acid-denatured Enolase (Sigma-Aldrich, St Louis, MO) and $50 \mu \mathrm{M}$ ATP at $30^{\circ} \mathrm{C}$ for $30 \mathrm{~min}$. The reaction was stopped by adding SDS sample-loading buffer and boiling. The reaction products were analyzed using western blot.

\section{Western blot analysis}

Proteins were fractionated with $10 \%$ SDS-polyacrylamide gel electrophoresis and transferred onto a nitrocellulose membrane (Bio-Rad, Hercules, CA, USA). The membrane was blocked with $5 \%$ BSA in Tris-buffered saline containing $0.05 \%$ Tween-20, and then incubated with primary antibodies. The primary antibodies were detected with horseradish peroxidase-conjugated second antibodies (Sigma-Aldrich). The antigen-antibody complex was visualized using a chemiluminescence detection kit (Roche, Indianapolis, IN, USA). The density of each protein band was scanned and the data were subjected to statistical analysis. Antiphosphotyrosine antibody was purchased from Cell Signaling (Danvers, MA, USA). Anti-EphA3 antibody was purchased from Santa Cruz Biotechnology.

\section{Cell migration assay}

For the wound-healing assay, $5 \times 10^{5}$ transfected 293A cells were seeded on fibronectin-coated dishes and cultured for 1 day. The cell monolayer was scratched with micropipette tips and images were captured at the indicated hours after wounding using a Nikon microscope. To quantify the results, cells expressing GFP with or without EphA3 that migrated into the gap were counted. For Transwell migration assay, $2 \times 10^{4}$ cells were re-plated onto the upper chamber of a Transwell filter with $8 \mu \mathrm{m}$ pores (Corning Inc Life Sciences, Wilkes-Barre, PA, USA) coated with $10 \mu \mathrm{g} /$ $\mathrm{ml}$ fibronectin underneath. After $16 \mathrm{~h}$, cells were fixed with $4 \%$ paraformaldehyde in PBS. Nonmigrated cells on the upper side of the filter were wiped off with a cotton swab. Transfected 293A cells were analyzed under a Nikon fluorescence microscope. Same amount of cells were plated onto fibronectin-coated plates in parallel to determine the number of cells used in the assays. Relative cell migration was determined by the number of migrated GFPpositive cells normalized to the total GFP-positive cells adhering to fibronectin-coated plates. For each experiment, the number of cells in five random fields on the underside of the filter was counted and at least six independent assays were performed.

\section{Neurite outgrowth and growth cone collapse assay}

Eph receptor-transfected NG108-15 cells were cultured at $37^{\circ} \mathrm{C}$ in DMEM plus $1 \mathrm{mM}$ of dibutyryl cyclic AMP (dbcAMP) with $2 \mu \mathrm{g} /$ $\mathrm{ml}$ cross-linked ephrin-A5, overnight. Cells were fixed with $4 \%$ paraformaldehyde in PBS and stained with Texas Red-conjugated phalloidin. Neurite outgrowth in NG108-15 cells was observed using photographs taken under a Nikon TE200 fluorescence microscope. Primary neurons were cultured for 2 to 3 days before ephrin-A5 treatment. Neurons were stimulated with cross-linked ephrin-A5, fixed with 4\% paraformaldehyde, and stained with Texas Red-conjugated phalloidin. Stained primary neurons were observed under a Zeiss Axiovert 200M fluorescence microscope. The total number of axons and the number of growth cone containing axons were enumerated. For transfected cultures, only GFPexpressing cells were included in the quantification.

\section{Statistical analysis}

For comparison of multiple groups, one-way ANOVA was used and followed by Bonferroni's post hoc test of selected pairs. The $\alpha$ level (0.05) used in post hoc analyses was adjusted by the number of comparisons (Bonferroni correction).

\section{Acknowledgment}

This research was supported in part by grants to RZ from National Science Foundation (US) (0548541), New Jersey Commission on Spinal Cord Research, and NIH (5P01HD023315).

\section{References}

1 Zhou R. The Eph family receptors and ligands. Pharmacol Ther 1998; 77:151-181.

2 Nakamoto M, Bergemann AD. Diverse roles for the Eph family of receptor tyrosine kinases in carcinogenesis. Microsc Res Tech 2002; 59:58-67.

3 Pasquale EB. Eph-ephrin bidirectional signaling in physiology and disease. Cell 2008; 133:38-52.

4 Zhou R. Regulation of topographic projection by the Eph family receptor Bsk (EphA5) and its ligands. Cell Tissue Res 1997; 290:251-259.

5 Flanagan JG, Vanderhaeghen P. The ephrins and Eph receptors in neural development. Annu Rev Neurosci 1998; 21:309345 .

6 McLaughlin T, O'Leary DD. Molecular gradients and development of retinotopic maps. Annu Rev Neurosci 2005; 28:327-355.

7 Wilkinson DG. Eph receptors and ephrins: regulators of guidance and assembly. Int Rev Cytol 2000; 196:177-244.

8 Kuijper S, Turner CJ, Adams RH. Regulation of angiogenesis by Eph-ephrin interactions. Trends Cardiovasc Med 2007; 17:145-151.

9 Zhang J, Hughes S. Role of the ephrin and Eph receptor tyrosine kinase families in angiogenesis and development of the 
cardiovascular system. $J$ Pathol 2006; 208:453-461.

10 Compagni A, Logan M, Klein R, Adams RH. Control of skeletal patterning by ephrinB1-EphB interactions. Dev Cell 2003; 5:217-230.

11 Davy A, Aubin J, Soriano P. Ephrin-B1 forward and reverse signaling are required during mouse development. Genes Dev 2004; 18:572-583.

12 Ivanov AI, Romanovsky AA. Putative dual role of ephrinEph receptor interactions in inflammation. IUBMB Life 2006; 58:389-394.

13 Cooper MA, Son AI, Komlos D, Sun Y, Kleiman NJ, Zhou R. Loss of ephrin-A5 function disrupts lens fiber cell packing and leads to cataract. Proc Natl Acad Sci USA 2008; 105: $16620-16625$.

14 Shiels A, Bennett TM, Knopf HL, et al. The EPHA2 gene is associated with cataracts linked to chromosome 1p. Mol Vis 2008; 14:2042-2055.

15 Zhang T, Hua R, Xiao W, et al. Mutations of the EPHA2 receptor tyrosine kinase gene cause autosomal dominant congenital cataract. Hum Mutat 2009; 30:E603-E611.

16 Klein R. Bidirectional modulation of synaptic functions by Eph/ephrin signaling. Nat Neurosci 2009; 12:15-20.

17 Heroult M, Schaffner F, Augustin HG. Eph receptor and ephrin ligand-mediated interactions during angiogenesis and tumor progression. Exp Cell Res 2006; 312:642-650.

18 Wimmer-Kleikamp SH, Lackmann M. Eph-modulated cell morphology, adhesion and motility in carcinogenesis. IUBMB Life 2005; 57: 421-431.

19 Wang X, Roy PJ, Holland SJ, Zhang LW, Culotti JG, Pawson T. Multiple ephrins control cell organization in C. elegans using kinase- dependent and -independent functions of the VAB-1 Eph receptor. Mol Cell 1999; 4:903-913.

20 Fang WB, Brantley-Sieders DM, Parker MA, Reith AD, Chen J. A kinase-dependent role for EphA2 receptor in promoting tumor growth and metastasis. Oncogene 2005; 24:7859-7868.

21 Tolias KF, Bikoff JB, Burette A, et al. The Rac1-GEF Tiam1 couples the NMDA receptor to the activity-dependent development of dendritic arbors and spines. Neuron 2005; 45:525538.

22 Taddei ML, Parri M, Angelucci A, et al. Kinase-dependent and -independent roles of EphA2 in the regulation of prostate cancer invasion and metastasis. Am J Pathol 2009; 174:14921503.

23 Yue Y, Chen ZY, Gale NW, et al. Mistargeting hippocampal axons by expression of a truncated Eph receptor. Proc Natl Acad Sci USA 2002; 99:10777-10782.

$24 \mathrm{Hu}$ Z, Yue X, Shi G, et al. Corpus callosum deficiency in transgenic mice expressing a truncated ephrin-A receptor. $J$ Neurosci 2003; 23:10963-10970.

25 Pawson T. Regulation and targets of receptor tyrosine kinases. Eur J Cancer 2002; 38 Suppl 5:S3-S10.

26 Pawson T. Specificity in signal transduction: from phosphotyrosine-SH2 domain interactions to complex cellular systems. Cell 2004; 116:191-203.

27 Lawrenson ID, Wimmer-Kleikamp SH, Lock P, et al. EphrinA5 induces rounding, blebbing and de-adhesion of EphA3expressing $293 \mathrm{~T}$ and melanoma cells by CrkII and Rhomediated signalling. J Cell Sci 2002; 115:1059-1072.

28 Zisch AH, Pazzagli C, Freeman AL, et al. Replacing two conserved tyrosines of the EphB2 receptor with glutamic acid prevents binding of $\mathrm{SH} 2$ domains without abrogating kinase activity and biological responses. Oncogene 2000; 19:177187.

29 Zisch AH, Kalo MS, Chong LD, Pasquale EB. Complex formation between EphB2 and Src requires phosphorylation of tyrosine 611 in the EphB2 juxtamembrane region. Oncogene 1998; 16:2657-2670.

30 Kalo MS, Yu HH, Pasquale EB. In vivo tyrosine phosphorylation sites of activated ephrin-B1 and ephB2 from neural tissue. J Biol Chem 2001; 276:38940-38948.

31 Knoll B, Drescher U. Src family kinases are involved in EphA receptor-mediated retinal axon guidance. J Neurosci 2004; 24:6248-6257.

32 Zimmer G, Kastner B, Weth F, Bolz J. Multiple effects of ephrin-A5 on cortical neurons are mediated by SRC family kinases. J Neurosci 2007; 27:5643-5653.

33 Yue X, Dreyfus C, Kong TA, Zhou R. A subset of signal transduction pathways is required for hippocampal growth cone collapse induced by ephrin-A5. Dev Neurobiol 2008; 68:1269-1286.

34 Egea J, Klein R. Bidirectional Eph-ephrin signaling during axon guidance. Trends Cell Biol 2007; 17:230-238.

35 Mann F, Miranda E, Weinl C, Harmer E, Holt CE. B-type Eph receptors and ephrins induce growth cone collapse through distinct intracellular pathways. J Neurobiol 2003; 57:323-336.

36 Wybenga-Groot LE, Baskin B, Ong SH, Tong J, Pawson T, Sicheri F. Structural basis for autoinhibition of the Ephb2 receptor tyrosine kinase by the unphosphorylated juxtamembrane region. Cell 2001; 106:745-757.

37 Deroanne C, Vouret-Craviari V, Wang B, Pouyssegur J. EphrinA1 inactivates integrin-mediated vascular smooth muscle cell spreading via the Rac/PAK pathway. J Cell Sci 2003; 116:1367-1376.

38 Dail M, Richter M, Godement P, Pasquale EB. Eph receptors inactivate R-Ras through different mechanisms to achieve cell repulsion. J Cell Sci 2006; 119:1244-1254.

39 Klee WA, Nirenberg M. A neuroblastoma times glioma hybrid cell line with morphine receptors. Proc Natl Acad Sci USA 1974; 71:3474-3477.

40 Davis TL, Walker JR, Loppnau P, Butler-Cole C, AllaliHassani A, Dhe-Paganon S. Autoregulation by the juxtamembrane region of the human ephrin receptor tyrosine kinase A3 (EphA3). Structure 2008; 16:873-884.

41 Goldshmit Y, McLenachan S, Turnley A. Roles of Eph receptors and ephrins in the normal and damaged adult CNS. Brain Res Brain Res Rev 2006; 52:327-345.

42 Tong J, Elowe S, Nash P, Pawson T. Manipulation of EphB2 regulatory motifs and $\mathrm{SH} 2$ binding sites switches MAPK signaling and biological activity. J Biol Chem 2003; 278:61116119.

43 Parri M, Buricchi F, Giannoni E, et al. EphrinA1 activates a $\mathrm{Src} /$ focal adhesion kinase-mediated motility response leading to rho-dependent actino/myosin contractility. J Biol Chem 2007; 282:19619-19628.

$44 \mathrm{Hu}$ T, Shi G, Larose L, Rivera GM, Mayer BJ, Zhou R. Regulation of process retraction and cell migration by EphA3 is mediated by the adaptor protein Nck1. Biochemistry 2009; 48:6369-6378. 
45 Fang WB, Brantley-Sieders DM, Hwang Y, Ham AJ, Chen J. Identification and functional analysis of phosphorylated tyrosine residues within EphA2 receptor tyrosine kinase. J Biol Chem 2008; 283:16017-16026.

46 Ito Y, Oinuma I, Katoh H, Kaibuchi K, Negishi M. Sema4D/ plexin-B1 activates GSK-3beta through R-Ras GAP activity, inducing growth cone collapse. EMBO Rep 2006; 7:704-709.

47 Oinuma I, Katoh H, Negishi M. Semaphorin 4D/Plexin-B1mediated R-Ras GAP activity inhibits cell migration by regulating beta(1) integrin activity. J Cell Biol 2006; 173:601-613.

48 Wang B, Zou JX, Ek-Rylander B, Ruoslahti E. R-Ras contains a proline-rich site that binds to $\mathrm{SH} 3$ domains and is required for integrin activation by R-Ras. J Biol Chem 2000; 275:5222-5227.

49 Hansen M, Prior IA, Hughes PE, et al. C-terminal sequences in R-Ras are involved in integrin regulation and in plasma membrane microdomain distribution. Biochem Biophys Res Commun 2003; 311:829-838.

50 Nakamura T, Komiya M, Sone K, et al. Grit, a GTPase-acti- vating protein for the Rho family, regulates neurite extension through association with the TrkA receptor and N-Shc and CrkL/Crk adapter molecules. Mol Cell Biol 2002; 22:87218734.

51 Huot J. Ephrin signaling in axon guidance. Prog Neuropsychopharmacol Biol Psychiatry 2004; 28:813-818.

52 Bardelli A, Parsons DW, Silliman N, et al. Mutational analysis of the tyrosine kinome in colorectal cancers. Science 2003; 300:949.

53 Balakrishnan A, Bleeker FE, Lamba S, et al. Novel somatic and germline mutations in cancer candidate genes in glioblastoma, melanoma, and pancreatic carcinoma. Cancer Res 2007; 67:3545-3550.

54 Ding L, Getz G, Wheeler DA, et al. Somatic mutations affect key pathways in lung adenocarcinoma. Nature 2008; 455:1069-1075.

55 Bae HJ, Song JH, Noh JH, et al. Low frequency mutation of the Ephrin receptor-A3 gene in hepatocellular carcinoma. Neoplasma 2009; 56:331-334.

Supplementary information is linked to the online version of the paper on the Cell Research website. 